Z Gerontol Geriat 2019 · 52:215-217 https://doi.org/10.1007/s00391-019-01552-0 Online publiziert: 30. April 2019

(c) Springer Medizin Verlag GmbH, ein Teil von Springer Nature 2019

Mit dem Themenschwerpunkt Wohnen im Alter bei lebenslanger Behinderung wird in der Zeitschrift für Gerontologie und Geriatrie ein Thema aufgegriffen, das sowohl in der Eingliederungshilfe als auch in der Gerontologie zunehmend Beachtung findet. Die Gründe hierfür lassen sich sowohl in demografischen Veränderungen als auch in gesellschaftlichen Entwicklungen verorten, die sowohl durch die Ratifizierung der UN-Behindertenrechtskonvention (UN-BRK) in Deutschland vor zehn Jahren als auch durch die Verabschiedung des Bundesteilhabegesetzes im Dezember 2016, mit dem die Umsetzung der UN-BRK weiter rechtlich verankert werden sollte, ihren Ausdruck finden. Ältere und alte Menschen mit einer (auch lebenslangen) Behinderung sind allerdings eine sehr heterogene Gruppe in Bezug auf ihre Lebenslagen, Ressourcen und Unterstützungsbedarfe, vergleichbar mit allen anderen Menschen in der Lebensphase Alter.

Aus den vorliegenden und wenig differenzierenden Daten wird deutlich, dass ihre Zahl steigt. Dem zweiten Teilhabebericht der Bundesregierung zufolge [1, S. $41 \mathrm{ff}$.] lebten im Jahr 2013 insgesamt 12,77 Mio. Menschen mit Beeinträchtigungen in Deutschland. Dies entspricht einem Anteil von 15,8\% an der Gesamtbevölkerung. Dabei steigt der Anteil von Menschen mit Beeinträchtigungen an der Gesamtbevölkerung mit zunehmendem Lebensalter deutlich an. Er beträgt $34 \%$ bei den 65 - bis 79 -Jährigen und

Sabine Kühnert ${ }^{1} \cdot$ Cornelia Kricheldorff $^{2}$

'Evangelische Hochschule Rheinland-Westfalen-Lippe, Bochum, Deutschland

${ }^{2}$ Katholische Hochschule Freiburg, Freiburg, Deutschland

\title{
Wohnen im Alter bei lebenslanger Behinderung - ein gerontologisches Thema mit wachsender Relevanz
}

$47,7 \%$ bei den ab 80 -Jährigen, wobei dies sowohl lebenslange als auch altersbedingt erworbene Beeinträchtigungen umfasst. Es überwiegen zwar körperliche Behinderungen und Sinnesbehinderungen, doch weisen auch $38 \%$ der 45 - bis 64 Jährigen und $9 \%$ der über 65-Jährigen nach der amtlichen Schwerbehindertenstatistik von 2013 eine sog. geistige Behinderung auf. Unter Berücksichtigung der steigenden Lebenserwartung von Menschen mit einer lebenslangen Behinderung [2] wird deutlich, dass eine differenzierte Beschäftigung mit ihren Lebenslagen und den damit verbundenen Unterstützungserfordernissen in der Gerontologie dringend geboten ist.

Die Auseinandersetzung mit der Lebenssituation älter werdender Menschen mit einer lebenslangen Behinderung muss dabei auch vor dem Hintergrund sich verändernder gesellschaftlicher Rahmenbedingungen und eines daraus resultierenden veränderten Selbstverständnis der Betroffen und ihrer An- und Zugehörigen stattfinden. Seit den 1970erJahren wird zunehmend der Anspruch auf Selbstvertretung und Selbstbestimmung eingefordert, der auf ein anderes Verständnis von Behinderung sowie ein selbstbestimmtes Leben und Akzeptanz in der Gesellschaft abzielt. Sowohl in der International Classification of Functioning, Disability and Health (ICF) als auch in der 2009 in Deutschland ratifizierten UN-BRK kommt dieses veränderte Selbstverständnis zum Ausdruck. Dieses betont die gesellschaftliche
Mitbedingtheit von Beeinträchtigungen und die daraus resultierende Exklusion und fordert die Gesellschaft unter dem Stichwort Inklusion zur Öffnung für Menschen mit einer Behinderung auf. Auch älteren Menschen mit einer lebenslangen Behinderung soll, trotz anderweitiger langjähriger Sozialisationserfahrungen in Spezialeinrichtungen, die Möglichkeit zur Teilhabe gegeben werden.

Dass dieser Anspruch insbesondere für ältere Menschen bislang noch kaum umgesetzt wird, hat mehrere Ursachen. Zum einen haben sie, in der Nachkriegszeit geboren, kaum eine schulische Förderung, dafür aber negative Stigmatisierungen erfahren und leben zum Großteil fast ihr gesamtes Leben in Komplexeinrichtungen. So weisen Zahlen für den Landschaftsverband Westfalen-Lippe aus, dass $42 \%$ aller Menschen mit einer sog. geistigen Behinderung in stationären Wohnformen leben. Für die Gruppe der über 50-Jährigen sind es sogar $60 \%$ [3]. Alternative Lebensformen sind ihnen kaum bekannt und die Fähigkeit zu Artikulation und Umsetzung eigener Lebensvorstellungen, beispielweise in Bezug auf Wohnwünsche, aufgrund dieser Sozialisationserfahrungen kaum ausgebildet und mitunter behinderungsbedingt auch schwer erfassbar. Hinzu kommt, dass es sich bei älteren Menschen mit einer lebenslangen Behinderung, insbesondere einer sog. geistigen Behinderung, um eine besonders vulnerable Gruppe handelt, die im 
Vergleich zu Gleichaltrigen ohne eine Behinderung über geringere finanzielle Ressourcen verfügt und eine schlechtere soziale Verankerung aufweist - dies als Folge fehlender Familiengründung und sozialer Beziehungen außerhalb der Einrichtung, in der sie leben. Entsprechend der „Environmental-docility“-Hypothese von Lawton [4] kommt deshalb der Gestaltung von Umweltbedingungen eine hohe Bedeutung zu, um den Betroffenen ein größtmögliches Ausmaß an Selbstbestimmung und Selbstständigkeit sowie die Umsetzung persönlicher Lebensvorstellungen $\mathrm{zu}$ ermöglichen.

In Artikel 19 der UN-BRK ist das Recht auf wunschgemäßes Wohnen und eine unabhängige Lebensführung verankert. Menschen mit einer Behinderung sollen ihren Aufenthaltsort frei wählen können und nicht verpflichtet sein, in besonderen Wohnformen zu leben, und sie sollen Zugang zu gemeindenahmen Unterstützungsdiensten haben.

Die beiden Projekte „Modelle für die Unterstützung der Teilhabe von Menschen mit geistiger Behinderung im Alter innovativ gestalten (MUTIG)", gefördert vom BMBF, und "Wahlmöglichkeiten sichern", gefördert von der Stiftung Wohlfahrtspflege, wollen durch eine explorative Wissensgenerierung einen Beitrag zur fundierten Umsetzung des Artikel 19 der UN-BRK leisten. Sie erfassen und beschreiben die Wohnsituationen für die Gruppe der Menschen mit einer sog. geistigen Behinderung und mit komplexen Behinderungen im Alter und prüfen die Möglichkeiten zur Realisierung von Wohnwünschen für diese Zielgruppe, die bisher kaum im Fokus der Gerontologie war. In den vier Beiträgen dieses Themenheftes werden relevante Teilergebnisse aus beiden Projekten vorgestellt, die sich schwerpunktmäßig auf quantitative Analysen zur Wohnsituation und auf qualitative Erhebungen zu Wohnwünschen und Umzugsentscheidungen beziehen.

Der erste Beitrag von Antonia Thimm, Friedrich Dieckmann und Theresia Haßler mit dem Titel „In welchen Wohnsettings leben ältere Menschen mit geistiger Behinderung? Ein quantitativer Vergleich von Altersgruppen für Westfalen-Lippe“ zielt mit einer durch- geführten Bestandserhebung auf eine Verbesserung des Wissensstandes zur Wohnsituation von älteren Menschen mit einer sog. geistigen Behinderung ab. Dazu wird auf Grundlage der Daten des Landschaftsverbandes Westfalen-Lippe sowie ergänzender Primärerhebungen in ausgewählten Pflegeeinrichtungen in Westfalen-Lippe die Wohnsituation von Erwachsenen mit einer geistigen Behinderung untersucht. Differenziert wird dabei nach Personen, die Leistungen der Eingliederungshilfe erhalten und ambulant oder stationär wohnen, und Personen, die in allgemeinen oder in speziellen Pflegeeinrichtungen leben. Die Ergebnisse verdeutlichen, dass sich ein Großteil der Erwachsenen mit einer geistigen Behinderung in stationären Wohnsettings der Eingliederungshilfe befindet, wobei mit zunehmendem Lebensalter ihr Anteil steigt. Die für die jüngeren Altersgruppen ebenfalls anzutreffenden Wohnformen eines selbstständigen Wohnens, entweder allein, mit Unterstützung durch Angehörige oder durch ambulante Dienste, sind bei älteren Menschen kaum anzutreffen. Stattdessen gewinnen Pflegeeinrichtungen nach SGB XI, mit ihren deutlich eingeschränkten Teilhabemöglichkeiten, mit zunehmendem Alter an Bedeutung und veranschaulichen die Benachteiligung dieser Personengruppe in Bezug auf eine möglichst selbstständige Lebensführung.

„Wohnwünsche von Menschen mit komplexer Behinderung" ist der Themenschwerpunkt des zweiten Beitrags von Karin Schrooten, Carina Bössing, Karin Tiesmeyer und Dieter Heitmann. Die Autorengruppe widmet sich einer besonders vulnerablen Zielgruppe, deren eingeschränkte Möglichkeiten zur Artikulation von Wohnwünschen und -bedürfnissen besondere methodische Zugangsweisen erfordern. Auch die Ergebnisse dieses Beitrags verdeutlichen, dass die Umsetzung des Artikel 19 UNBRK für die gewählte Zielgruppe kaum erkennbar ist. Umzüge werden, wie auch die Ergebnisse des ersten Beitrags vermuten lassen, aufgrund eines steigenden Pflegebedarfs durchgeführt und nicht als Ergebnis von Entscheidungsprozessen der Betroffenen. Die von den Autoren entwickelten und in exemplarischen Fallstudien erprobten Verfahren zur Ermittlung von Wohnwünschen zeigen hingegen eine Perspektive auf, wie Menschen mit komplexen Behinderungen mehr Autonomie und Selbstbestimmung in der Gestaltung ihrer Wohnsituation erhalten können.

Dass Umzüge bei Menschen mit einer sog. geistigen Behinderung kaum stattfinden und wenn, dann überwiegend in eine stationäre Pflegeeinrichtung, bestätigen auch die Ergebnisse von Theresia Haßler, Antonia Thimm und Friedrich Dieckmann zum Thema „Umzüge von älteren Menschen mit geistiger Behinderung - eine quantitative Analyse“. Auch diesen Ergebnissen liegen - wie auch im ersten Beitrag - Daten des Landschaftsverbandes Westfalen-Lippe zugrunde, die im Hinblick auf stattgefundene Umzüge ausgewertet werden. Es wird deutlich, dass nur wenige ältere Menschen mit sog. geistiger Behinderung überhaupt umziehen und wenn, dann vielfach aus einer stationären Einrichtung der Eingliederungshilfe in ein Pflegeheim nach SGB XI. Nur ein geringer Anteil wechselt aus einer stationären Einrichtung in ein ambulant betreutes Wohnsetting. Wie die Autoren betonen, können diese Daten zwar Veränderungen in der Wohnsituation abbilden, jedoch keine Schlussfolgerungen zu den Umzugsgründen erlauben, aus denen sich möglicherweise Anforderungen an die Gestaltung der einzelnen Wohnangebote ableiten lassen.

Diese Entscheidungsprozesse versuchen Friedrich Dieckmann, Bianca Rodekohr und Christin Mätze mit ihrem Beitrag „Umzugsentscheidungen in Pflegeeinrichtungen bei älteren Menschen mit geistiger Behinderung" zu skizzieren. Aus den von ihnen durchgeführten Fallstudien in speziellen Pflegeeinrichtungen wird deutlich, dass Umzugsentscheidungen von den Bewohnern kaum selbstbestimmt als Ergebnis vorheriger Beratung und Information getroffen wurden. Stattdessen werden sie überwiegend aus institutioneller Notwendigkeit veranlasst, wenn aus Sicht der Pflegeeinrichtung der steigende Pflegebedarf nicht mehr gedeckt werden kann. Damit zeigen die Ergebnisse einen konkreten 
Handlungsbedarf zur Veränderung von Versorgungsanboten und Prozessen der Entscheidungsfindung auf, um den Ansprüchen auf Selbstbestimmung und Teilhabe der weiter ansteigenden Anzahl älter werdender Menschen mit einer lebenslangen Behinderung zukünftig besser gerecht zu werden.

Insgesamt zeigt sich ein sehr defizitäres Bild in Bezug auf die Achtung individueller Wohnbedürfnisse und das Recht auf Autonomie bei der Wahl der Wohnform für die Gruppe der älteren Menschen mit einer lebenslangen Beeinträchtigung, speziell bei geistiger Behinderung. Die strukturellen Rahmenbedingungen in der einschlägigen Fachpraxis schränken dieses Recht bislang eindeutig ein. Weder wurden bisher die erforderlichen leistungsrechtlichen Grundlagen geschaffen, v.a. für ältere Menschen mit komplexen Behinderungen, noch sind Mitarbeiter und Träger hinreichend sensibilisiert und qualifiziert, mögliche Alternativen zur aktuellen Wohnsituation gemeinsam mit den Betroffenen zu erarbeiten und so umzusetzen, wie es die UN-BRK fordert. Wissensdefizite über die unterschiedlichen Lebenssituationen von Menschen mit einer lebenslangen Behinderung, ihren jeweiligen Unterstützungsbedarf und Schwierigkeiten mit der Erfassung von Wohnwünschen bei Menschen mit Kommunikationsbeeinträchtigungen tragen dazu bei, dass beispielsweise die Umsetzung des Artikel 19 UN.BRK v.a. für ältere Menschen bislang kaum gelungen ist.

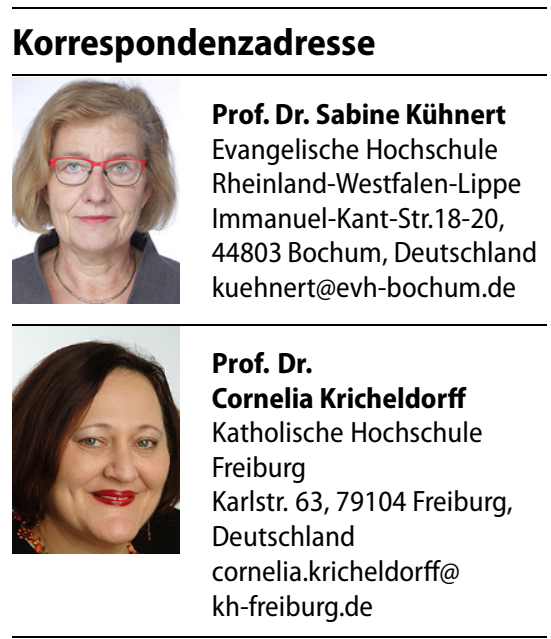

Interessenkonflikt. S. Kühnert und C. Kricheldorff geben an, dass kein Interessenkonflikt besteht.

\section{Literatur}

1. Bundesministerium für Arbeit und Soziales (Hrsg) (2016) Zweiter Teilhabebericht der Bundesregierung über die Lebenslagen von Menschen mit Beeinträchtigungen. Bundesministerium für Arbeit und Soziales, Bonn

2. Giovis C, Dieckmann F (2013) Ermittlung der Überlebenswahrscheinlichkeit bei Menschen mit geistiger Behinderung. In: Dieckmann F, Metzler $\mathrm{H}$ (Hrsg) Alter erleben - Lebensqualität und Lebenserwartung von Menschen mit geistige Behinderung im Alter. Abschlussbericht Katholische Hochschule Nordrhein-Westfalen, Abteilung Münster, Universität Tübingen, Z.I.E.L, S 128-163

3. Thimm A, Rodekohr B, Dieckmann F, Haßler Th (2018) Wohnsituation Erwachsener mit geistiger Behinderung in Westfalen-Lippe und Umzüge im Alter. Katholische Hochschule NRW, Münster (Erster Zwischenbericht zum Forschungsprojekt „Modelle für die Unterstützung der Teilhabe von Menschen mit geistiger Behinderung im Alter innovativ gestalten (MUTIG)")

4. Lawton MP (1982) Competence, environmental press and the adapation of older people. In: Lawton MP, Windley PG, Byerts ThO (Hrsg) Aging and the environment. Springer, New York, S33-59
Jetzt bewerben: DGG vergibt zwei Stipendien für die European Academy for Medicine of Ageing (EAMA)

Die Teilnahme am PostgraduiertenKolleg der European Academy for the Medicine of Ageing ist eine einzigartige Möglichkeit wissenschaftliche Kompetenzen im Bereich der Geriatrie zu erwerben und auf internationalem Niveau geriatrischwissenschaftliches Networking zu betreiben.

Die EAMA, die sich selbst als eine Institution sieht, welche die Kompetenzen junger Geriater, die eine Führungsposition einnehmen könnten, fördern und weiterentwickeln möchte, bietet neben Projektmanagement, Leadership-Training und Kommunikation eine hervorragende Möglichkeit zur Karriereentwicklung und eine exzellente Grundlage für internationales Networking.

Die Deutsche Gesellschaft für Geriatrie (DGG) vergibt zwei Vollstipendien, die die kompletten Kurskosten abdecken. Auf die Stipendien können sich geriatrisch tätige Ärzte, mit Interesse an Forschung und Lehre in der Geriatrie und der Bereitschaft, ein wissenschaftliches Netzwerk aufzubauen, bewerben.

Bitte bewerben Sie sich umgehend, die Benachrichtigung der geförderten Antragsteller erfolgt zum 01.07.2019. Die Stipendien werden nach Prüfung der Fördervoraussetzung vergeben. Das Executive Board der EAMA und der Vorstand der DGG werden die Auswahlentscheidung gemeinsam treffen.

Bitte richten Sie Ihre aussagekräftige Bewerbung mit einem formlosen Antrag sowie Ihrer Vorstellung der zukünftigen Kooperation im Science Network und der DGG per E-Mail mit dem Betreff „Stipendium EAMA" an die Geschäftsstelle der DGG: geschaeftsstelle@dggeriatrie.de oder reichen Sie Ihre Unterlagen auf dem Postweg ein: Geschäftsstelle der DGG, Seumestr.8, 10245 Berlin

Weiterführende Informationen zur EAMA finden Sie auf der EAMA-Website (www.eama.eu). 